\title{
Analysis of Optical Injection on Red and Blue Laser Diodes for High Bit-rate Visible Light Communication
}

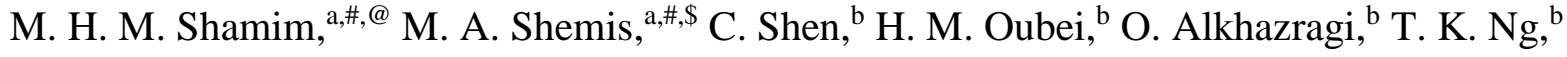 \\ B. S. Ooi, ${ }^{b}$ and M. Z. M. Khan ${ }^{\mathrm{a}, *}$ \\ ${ }^{a}$ Optoelectronics Research Laboratory, Electrical Engineering Department, King Fahd University of Petroleum \& Minerals, \\ Dhahran 31261, Saudi Arabia \\ ${ }^{b}$ Photonics Laboratory, Computer, Electrical and Mathematical Sciences and Engineering (CEMSE) division, King Abdullah \\ University of Science \& Technology (KAUST), Thuwal 23955-6900, Saudi Arabia \\ ${ }^{\#}$ Both authors contributed equally to this work \\ *Corresponding author: zahedmk@kfupm.edu.sa
}

\begin{abstract}
In this work, self-injection and external-injection in $\sim 450 \mathrm{~nm} \mathrm{InGaN/GaN} \mathrm{blue} \mathrm{and} \sim 650 \mathrm{~nm}$ $\mathrm{InGaP} / \mathrm{AlGaInP}$ red diode lasers are investigated. A distinct locking characteristic is observed in the selfinjection case with small $19 \mathrm{~cm}$ cavity length, demonstrating enhanced $\sim 2.34$ and $\sim 2.07 \mathrm{GHz} 3-\mathrm{dB}$ bandwidths, corresponding to a factor of $\sim 1.4$ and $\sim 1.1$ improvement, and reduced $\sim 60$ and $\sim 80 \mathrm{pm}$ spectral linewidths, for the blue and the red lasers, respectively. Moreover, this short external cavity self-injection locked system exhibited superior performance by a factor of 1.1-1.3 compared to the long cavity $(26 \mathrm{~cm})$ configuration. Conversely, the external optical injection exhibited weak locking signature with improved linewidths by a factor of $\sim 1.6-2.8$ and reaching as small as $\sim 70$ and $\sim 87 \mathrm{pm}$ for the blue laser, respectively, while almost doubling in the peak powers. Later, on-off keying modulation technique based data transmission rates of up to 3.5 and $4.5 \mathrm{~Gb} / \mathrm{s}$ are demonstrated on free-running blue and red laser diodes, respectively, employing an in-house laser diode mount based system. Moreover, owing to the bandwidth limitation of the optically injected systems, successful transmission of up to $2 \mathrm{~Gb} / \mathrm{s}$ is demonstrated with better performance compared to the respective free-running cases, in particular, the external-optically injected system demonstrated more than double improvement in the bit-error-rate.
\end{abstract}

Keywords: self-injection locking, external optical injection, visible light communication, semiconductor laser diodes.

\section{Introduction}

Recently, visible light communication (VLC), an alternative form of indoor optical wireless communication, attracted significant research focus as a complementing substitution for near-infrared (NIR) and radio frequency (RF) communication to meet the demand of high data rate communication at the user end [1]. The VLC technology potentially relieves the load on these networks [2] at the last mile access while possessing several advantages such as network security, utilization of unlicensed spectrum, low power consumption, the nonexistence of electromagnetic interferences, etc. Thus, VLC is now finding niche applications inside buildings [3], airplanes [4], traffic [5], satellite [6], and for underwater communication [7].

Initially, visible light emitting diodes (LEDs) were appealing light sources and acquired attention for VLC with the demonstration of Li-Fi technology [8]. However, their intrinsic characteristics such as efficiency droop, slow response time (due to long spontaneous radiative lifetime), and large parasitic capacitance, pose challenges by restricting their modulation bandwidth to few hundreds of $\mathrm{MHz}$ [9], [10]. As a result, a data rate of the VLC link was limited to $\mathrm{Mb} / \mathrm{s}$, for example, 150-Mb/s from a red LED using intensity

\footnotetext{
${ }^{\circledR}$ Currently with the Department of Electrical and Computer Engineering, McGill University, Montreal, QC, H3A OE9, Canada

${ }^{\$}$ Currently with the Department of Electrical and Computer Engineering, University of British Columbia, Vancouver, BC, V6T 1Z4, Canada
} 
modulation - direct detection method with on-off keying (OOK) [11]. Furthermore, spectrally efficient modulation techniques, e.g., orthogonal frequency division multiplexing (OFDM) and discrete multi-tone (DMT) were also employed with data rate improvements up to $3 \mathrm{~Gb} / \mathrm{s}$ for LED based on phosphor-coated single emission, and $3 \mathrm{~Gb} / \mathrm{s}$ for RGB LED [12]. Alternatively, faster LEDs such as resonant-cavity light emitting diodes (RCLEDs) or $\mu$ LED [11] have been proposed in VLC to mitigate the small modulation bandwidths of the conventional $p-i-n$ heterostructure LEDs, yet their optical power is relatively low.

On the other front, laser diodes are being considered as another promising source in VLC, recently, exhibiting superiority over LEDs due to their coherency and high power, which facilitates reaching higher transmission rates and longer distances [13]. For instance, using OOK [14] and 64-QAM OFDM [15], VLC communication achieved $2.5 \mathrm{~Gb} / \mathrm{s}$ and $9 \mathrm{~Gb} / \mathrm{s}$ data rates, respectively in the blue color region. Moreover, the demonstration of white light communication was also possible after using a phosphor in front of a blue laser diode using 16-QAM OFDM modulation scheme to achieve $4 \mathrm{~Gb} / \mathrm{s}$ data rate[1]. To further improve the laser diode characteristics, modulation bandwidth and particularly linewidth, very recently, optical injection assisting schemes, which is quite mature in the near-infrared regime, have been implemented at visible wavelengths [16]-[18]. In literature, two types of injection techniques have been reported. One of them called external injection locking (EIL) where an external laser known as the master laser is used to inject photons to another laser called slave laser to increase the modulation bandwidth. The other scheme uses a reflector to feedback part of the optical power into the laser itself, thus known as self-injection locking (SIL). So far, data communication on EIL based visible transmitters has been investigated in, [19], [20], and [21] where a modulation bandwidths improvement by 2.4, 4.5, and 5 times have been reported, and achievement of 20,16 , and $56 \mathrm{~Gb} / \mathrm{s}$ data rate using higher modulation schemes were possible. However, not much insight into the external optical injection (EOI) characterization has been performed, to the author's knowledge, except on an ultra-violet laser [22] wherein the effect of injection current on the locking characteristics was performed. Hence it necessities attention for further system optimization and eventual high bit-rate VLC. On the other hand, SIL on visible laser diodes has been reported to enhance the modulation bandwidths [23]. However, data communication on SIL based visible transmitter is yet to be investigated, to our knowledge.

In this work, we report the comprehensive analysis of external optical injection (EOI) on blue and red laser diodes, showing signatures of locking behavior. In particular, the effect of external optical feedback power on the laser linewidth, peak power, and stability are analyzed. Besides, we characterized SIL on these laser diodes in a short $19 \mathrm{~cm}$ external cavity free-space configuration and shed insight into the effect of external cavity length on the SIL characteristics by comparing with our recent results with long 26 and $56 \mathrm{~cm}$ cavity lengths. Thereafter, indoor OOK transmission experiments on $30 \mathrm{~cm}$ channel length, a better error-free transmission of up to $1.8 \mathrm{~Gb} / \mathrm{s}$ have been reported from the EOI red transmitter while a slightly improved transmission performance is demonstrated on SIL blue and red laser diodes at $2 \mathrm{~Gb} / \mathrm{s}$ compared to the freerunning counterparts. In all, this comprehensive study of optical injection on visible laser diode would provide guidance on further optimization of the respective systems in high bit-rate VLC.

\section{Self-injection Locked Blue and Red Laser Diodes}

\subsection{Experimental Setup}

Fig. 1 (a) shows the experimental setup of the self-injection locking scheme in the blue and red laser diode. In this experiment, commercial blue (Osram PL450B) and red (Sanyo DL6148-030) laser diodes have been used as the optical sources on a standard $1 \mathrm{GHz}$ bandwidth laser diode mount (Thorlabs LDM9T) [24]. A silver-coated mirror (Thorlabs PF10-03-P01) with 97.5\% reflectivity at $19 \mathrm{~cm}$ apart, forming an external cavity, is used to enable optical feedback to the source. A 92:8 \% (Transmission: Reflection) beam splitter 
is placed in the external cavity to ensure $8 \%$ of the light beam to reach the photodiode (PD) for communication and diagnostic purposes while $92 \%$ reflects into the diode for locking purpose. This insures reaching high injection ratio (IR, the ratio of the feedback power to laser diode power calculated at the laser front facet) values. A $30 \mathrm{~cm}$ VLC link is established from the optical source to a high-speed and lesssensitive $25 \mathrm{GHz}$ bandwidth photodiode (Alphalas UPD-50-UP) for the characterization purpose and a highly sensitive but low bandwidth $1.0 \mathrm{GHz}$ avalanche photodiode (APD, Menlo systems APD 210) for the communication experiments. Lenses are used for collimating (Lens 1, Thorlabs A110TM-A, focal length, $f=6.24 \mathrm{~mm}$ ) and focusing purpose (Lenses 1 and 2 (Thorlabs LB1471, $f=50 \mathrm{~mm}$ ), and 3 (Thorlabs LA4148, $f=50.2 \mathrm{~mm})$ ). For the transmission experiment, the laser diode is directly modulated with on-off keying (OOK) non-return to zero (NRZ) signal generated from a pattern generator to establish a VLC link between the source and the receiver. Then the received signal is analyzed using a digital communication analyzer (DCA, Agilent DCA-J 86100C) and the bit-error-rate tester (BERT, Agilent Technologies N4903B). For optical characterization, the emission spectrums are measured and recorded form the optical spectrum analyzer (OSA, Yokogawa AQ6373B) with $0.02 \mathrm{~nm}$ resolution, which is placed after the Lens 3.
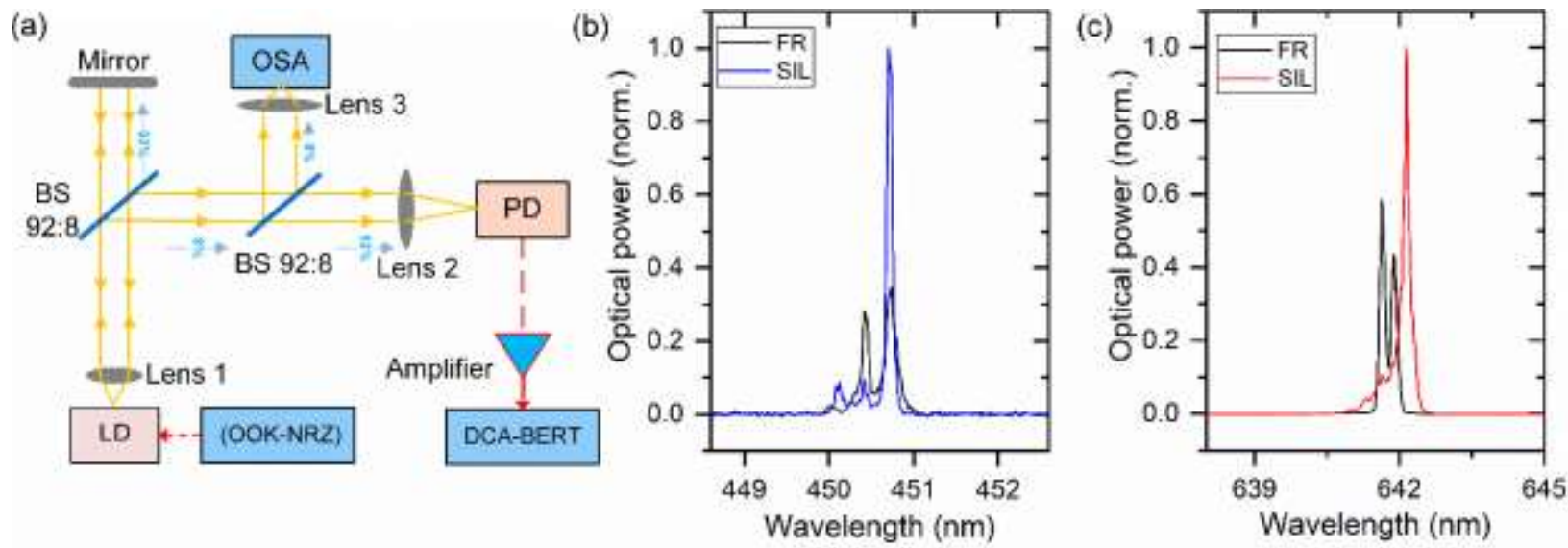

Figure 1: (a) Block diagram of self-injection locked laser system with integrated $30 \mathrm{~cm}$ VLC link for OOK modulation scheme. Comparison of the optical lasing spectrums of (b) blue and (b) red laser diodes in free-running (FR) and self-injection locked (SIL) case. The injection current for (c) and (d) are $40 \mathrm{~mA}$ and $110 \mathrm{~mA}$, respectively.

\subsection{Self-injection Locking Characterization}

The optical injection phenomenon creates a change in the laser gain threshold $g_{i n j}$, which depends on the free-running threshold gain $g_{t h}$, optical injection coefficient $C_{E}$, and the phase of the feedback injection beam, $\phi_{i n j}[25]$, given by:

$$
g_{i n j}=g_{t h}-L^{-1} C_{E} \cos \phi_{i n j}
$$

where $L$ is the laser cavity length with a roundtrip time $\tau_{L}$. This reduction in $g_{i n j}$ could be controlled by varying the external cavity length $L_{\text {ext }}$ (or the round trip delay $\tau_{i n j}$ ) while satisfying the phase matching condition, i.e. the phase of ,the external cavity injection beam mode, is resonant with the phase of a particular laser diode mode. This enables locking of that specific laser mode whose threshold gain reduces according to Eqn. (1), thus reaching the lasing condition first and hence exhibiting high power while suppressing the neighboring modes. The comparison of such single locked mode of the blue and red laser diode at a bias current of $40 \mathrm{~mA}$ and $110 \mathrm{~mA}$, respectively, and at $20^{\circ} \mathrm{C}$ room temperature, with its free running (FR) counterpart, is shown in Figure 1 (b) and (c). A substantial improvement of $\sim 2.8$ and $\sim 1.7$ times in the peak power of the blue and red lasers, respectively, were observed. Next, in Fig. 2, we 
systematically analyzed the self-injection locking behavior as a function of injection current of the laser diode. Figure 2(a) and (d) shows considerably enhancement in the measured modulation bandwidth of both the lasers along with their FR results. This observation essentially strengthens the successful locking phenomenon since the relaxation oscillation frequency $\widetilde{\omega}_{R}$, which dictates the modulation bandwidth $\left(f_{3 d B}\right)$, increases in the presence of optical feedback from that of FR case $\omega_{R}$, according to [26]:

$$
f_{3 d B}=\frac{1.55 \widetilde{\omega}_{R}}{2 \pi}=\frac{1.55 \omega_{R}}{2 \pi} \beta
$$

Here, $\beta$ is an additional term resulting from optical feedback and depends on the linewidth enhancement factor of the laser, $\phi_{i n j}, C_{E}, \tau_{L}$ and $\tau_{i n j}$, more details of which could be found in reference [25]. Hence, under injection locking $\beta$ is usually $>1$, thus enhancing the modulation bandwidth of the laser considerably [19], [21]. Now, referring back to Fig. 2, note that the modulation bandwidth initially improves with the increasing bias current and later at higher injections attains a steady state. The bandwidth of blue (red) laser diode increases to $\sim 2.34$ ( 2.07) $\mathrm{GHz}$ from $\sim 1.7$ (1.97) $\mathrm{GHz}$ at 70 (60) $\mathrm{mA}$ and injection current, representative of $\sim 1.4(\sim 1.1)$ times improvement. On the other hand, the spectral linewidth of the lasers at SIL condition, as shown in Figure 2(b) and (e), exhibits a smaller and stable value of $\sim 100 \mathrm{pm}$ till $110 \mathrm{~mA}$ for blue and $100 \mathrm{~mA}$ for red laser diodes compared to FR case. Thanks to injection locking condition, which also assisted in spectral linewidth reduction and for maximum gain condition, given by [25]:

$$
\Delta v_{i n j}=\frac{\Delta v_{t h}}{\left(1+\kappa \tau_{i n j} \tau_{L}^{-1}\right)^{2}}
$$

Where $\Delta v_{i n j}\left(\Delta v_{t h}\right)$ correspond to the linewidth with (without) optical feedback. Hence, under locking conditions, a smallest linewidth of $\sim 80 \mathrm{pm}$ for blue and $\sim 60 \mathrm{pm}$ for red laser diodes at 30 and $50 \mathrm{~mA}$ is noted which are $\sim 6.4$ and $\sim 1.65$ times smaller than the FR case. The linewidth of SIL laser diode compared to the FR case remained smaller and maintained a similar variation with respect to the bias current in both the lasers at all injections, suggesting an efficient locking at all injection currents. Referring to Figure 2(c) and (f), which plots the SMSR (the peak power of the locked mode to the highest peak power of the neighboring unlocked mode), a maximum value of $8.5 \mathrm{~dB}$ is observed at $30 \mathrm{~mA}$ for blue laser, which is almost double that of the FR case. Besides, improvement of SMSR is evident for all the injection currents and the maximum improvement of $\sim 7.0$ times is observed at $60 \mathrm{~mA}$ bias current. However, a steady mean value of $\sim 7 \mathrm{~dB}$ SMSR is measured from SIL red laser diode that is found to be more stable than the FR case, which kept changing arbitrarily across the entire injection, suggesting a stable system via SIL method although not much improvement in SMSR is noticed. We firmly believe a further proper optimization of the system via employing piezo stages to tune the external cavity should enable better SMSR for the system. Overall, better improvement in the performance of the blue laser is observed via self-injection locking compared to the red laser diode. We postulate that this difference of the performances is partially due to the material quality and device performance in InGaP/AlGaInP based laser is better than GaN/InGaN based $\mathrm{LD}$ as the latter being a comparatively matured material system. 
(a)

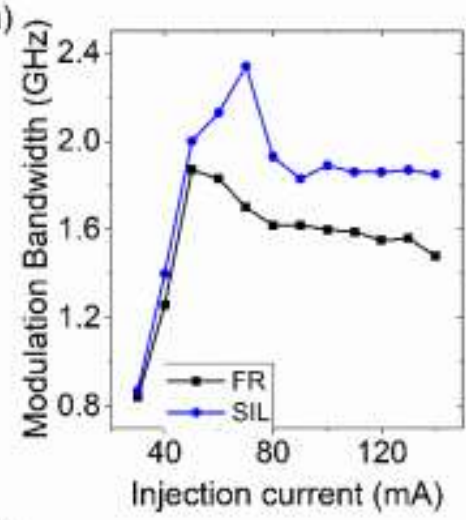

(d)

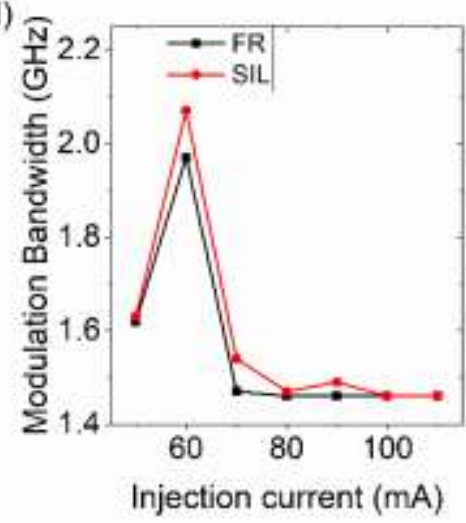

(b)

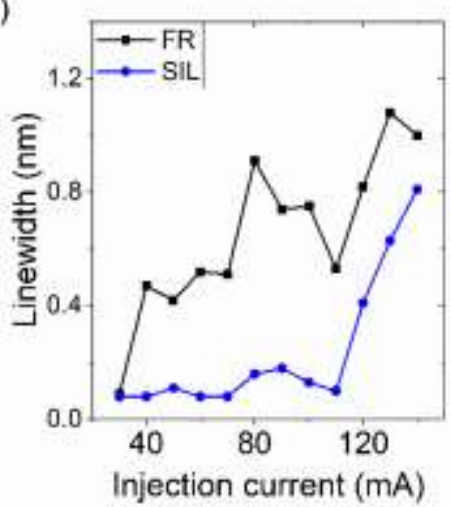

(e)

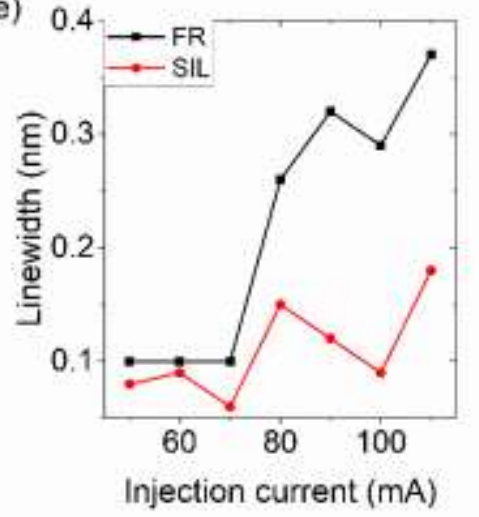

(c)

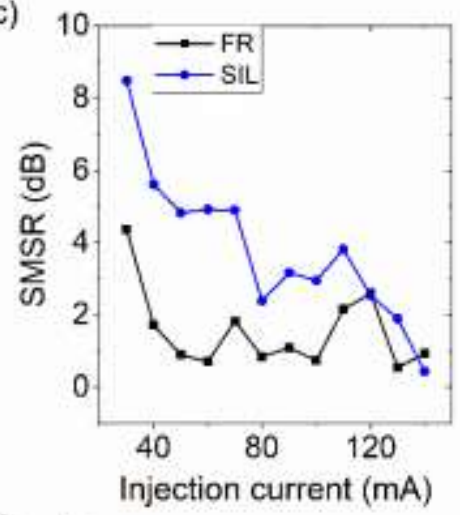

(f)

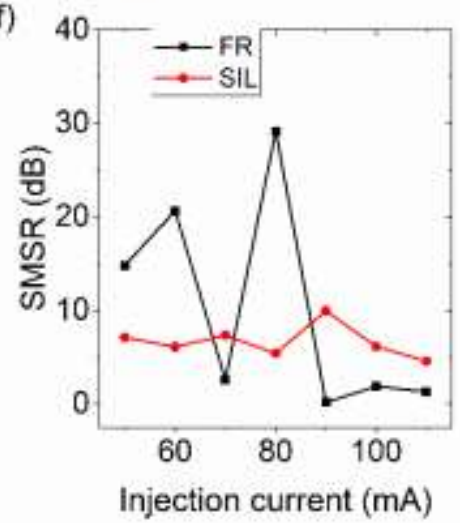

Figure 2. (a) and (d) Modulation bandwidth, (b) and (e) spectral linewidth, and (c) and (f) SMSR, as a function of current injection for blue (top row) and red (bottom row) laser diodes. The FR (black color) results are also plotted alongside self-injection locked counterpart (colored) in each case. The estimated injection ratio (IR) for blue and red laser diodes are fixed at $-0.7 \mathrm{~dB}$ and $-1.25 \mathrm{~dB}$, respectively.

Lastly, we compared the performance of the SIL system at different cavity length and summarized the results in Fig. 3. We have selected an injection current, $70 \mathrm{~mA}$ for both the lasers, at which the maximum bandwidth is observed in the blue laser diode (Figure 2 (a)), and outlined the other two parameters at the same injection, as shown in Figure 2 (b) and (c), respectively. Considering, the blue laser diode, a clear improvement in modulation bandwidth, spectral linewidth and the SMSR is evident from the Figure. 3 compared to the FR case, and at the comparatively shorter external cavity length. An enhanced bandwidth of $\sim 2.3 \mathrm{GHz}$, a linewidth of $\sim 80 \mathrm{pm}$ and SMSR of $\sim 4.9 \mathrm{~dB}$ is exhibited by $19 \mathrm{~cm}$ cavity length. However, the performance degrades at longer cavity length of $26 \mathrm{~cm}$ with corresponding values of $\sim 2.2 \mathrm{GHz}, \sim 100$ pm and $\sim 3.5 \mathrm{~dB}$, showing $\sim 1.4, \sim 6.25, \sim 2.7$ times improvement, respectively. On the other hand, the red laser diode, in general, showed improved performance at shorter cavity length with $\sim 60 \mathrm{pm}$ spectral linewidth and $7.5 \mathrm{~dB}$ SMSR, which are better than the long cavity results. This is ascribed to increased IR at shorter cavity length due to less scattering losses along with the free space compared to longer cavity length. This facilitates improved injection efficiency of the system and hence the performance. The estimated IR of $19 \mathrm{~cm}$ cavity is $\sim 0.7(\sim 1.3) \mathrm{dB}$ compared to $-1.3(-2.2) \mathrm{dB}$ at $26 \mathrm{~cm}$, for the blue (red) laser diode, which is approximately two times enhancement. On close observation of Figure 3, the red laser diode showed abrupt performance change with cavity length while the blue laser diode displayed the opposite behavior. This could be possible due to different material systems as well as the various degree of optimization of the SIL system. Since the blue laser diode exhibits multi-longitudinal mode emission characteristics, the system optimization is found to be easier with the manual tuning of the cavity length. 
Conversely, the red laser diode exhibited a comparatively narrow emission spectrum with dual or at most triple modes, hence was difficult to lock a particular mode using manual tuning. Utilizing a motorized piezo stage should enable proper optimization of the system with even better performances from both the lasers. Furthermore, the system is found to be very stable at this $19 \mathrm{~cm}$ cavity length, as reported earlier for the 26 and $56 \mathrm{~cm}$ cavities.

(a)

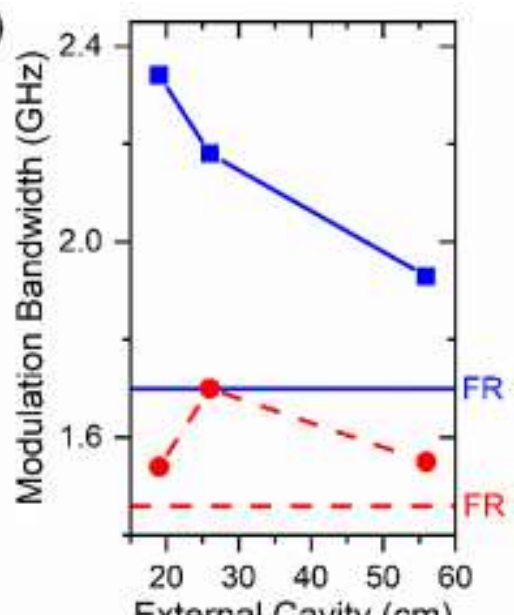

(b)

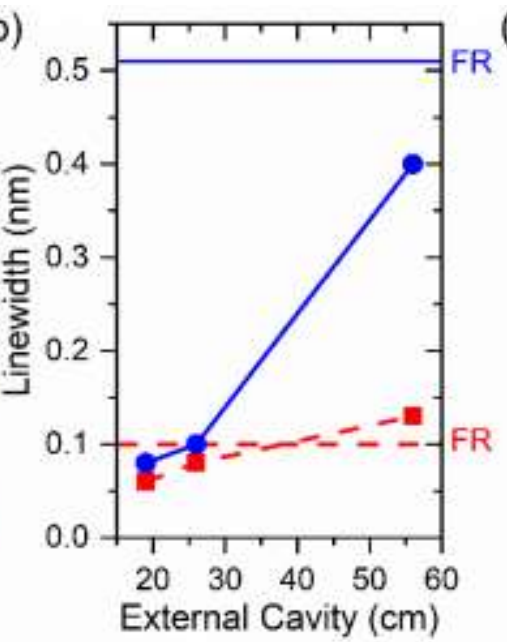

(c)

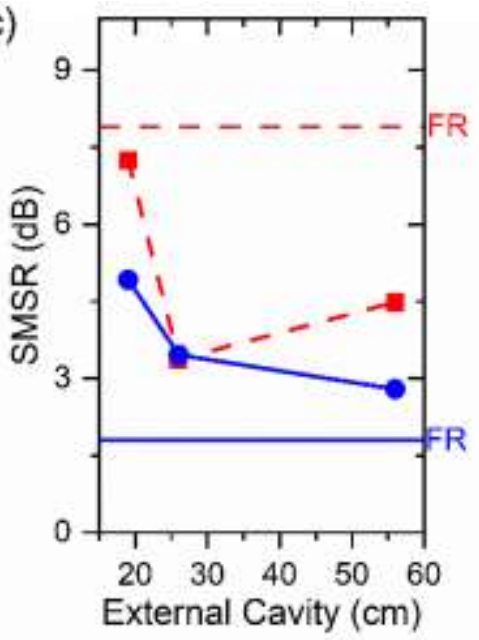

Figure 3: Comparison of (a) modulation bandwidth, (b) spectral linewidth, and (c) SMSR between the FR and self-injection locked blue and red TO can laser diode, which is obtained at different injection currents. The dashed and solid straight horizontal line corresponds to the respective FR laser case. In general, a considerable improvement in the bandwidth, linewidth, and SMSR is exhibited by SIL laser diodes.

\section{External Optical Injection in Blue and Red Laser Diodes}

\subsection{Experimental setup}

Figure 4 (a) shows the experimental setup of the EOI system. Two laser diodes of each type, one working as a master and other as a slave laser source, are separately mounted on a standard $1 \mathrm{GHz}$ standard laser diode mount (Thorlabs LDM9T) with an externally controlled integrated thermoelectric cooler. Two convex lenses 1 and 2 are deployed in front of these laser diodes to collimate and focus the beams. The master laser beam is guided and coupled into the slave laser diode via 92:8 \% beam splitter (BS1). The 92\% optical power of the master laser is used to lock the slave laser, to achieve high injection ratio, and the other $8 \%$ as the diagnostic beam as well as for communication purpose. As seen from Figure 4 (a), the $8 \%$ of the optical power is split further by a 50:50 \% beam splitter BS2 and sent to OSA and a high-speed photodiode to simultaneously study the characterization and communication performance. In this case, the VLC link is $30 \mathrm{~cm}$ long, formed between the source and the photodiode, and the transmission experiments are performed by directly modulating the slave laser diode by OOK-NRZ signal generated by a pattern generator at the transmitter side and APD at the receiving side followed by a DCA and BERT. 
(a)

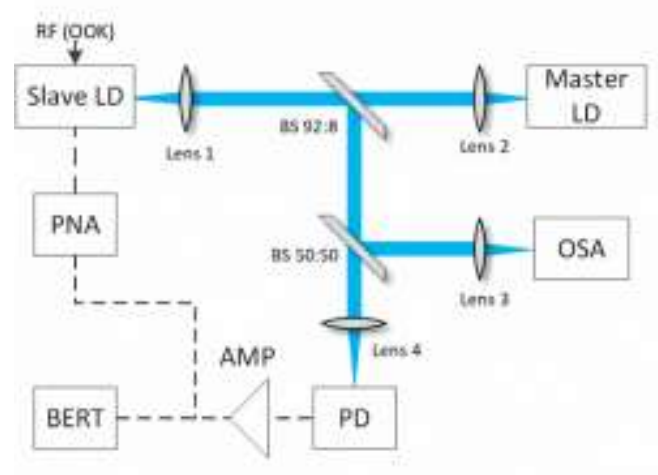

(b)

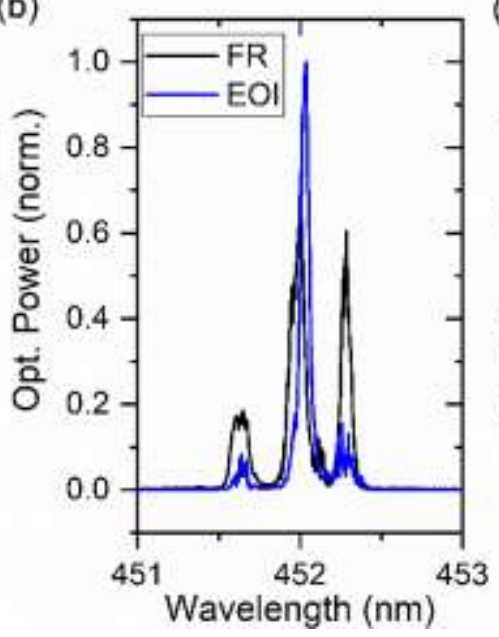

(c)

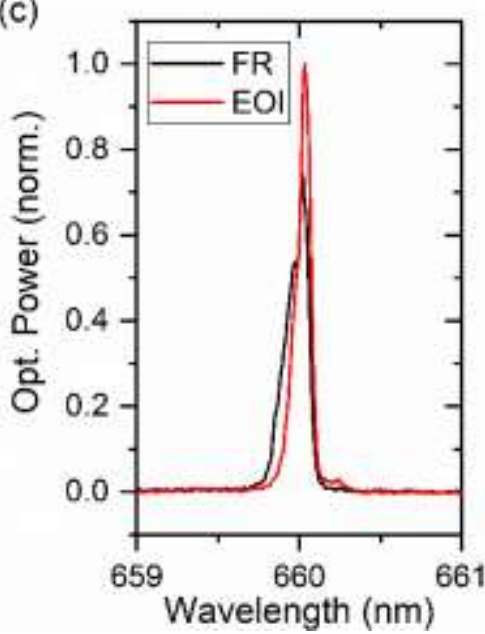

Figure 4: (a) Schematic diagram of external optical injection setup with $30 \mathrm{~cm}$ VLC link for OOK-NRZ modulation scheme. Comparison of the optical lasing spectrums of (b) blue and (c) red laser diodes in FR (FR) and external optical injection (EOI) case. The bias currents are (b) 68 mA and $61 \mathrm{~mA}$ and (c) $108 \mathrm{~mA}$ and $100 \mathrm{~mA}$ for the master and slave laser diodes, respectively.

\subsection{External Optical Injection Characterization}

Figure 4(b) and (c) shows a typical lasing spectrum of the blue and red laser diode, respectively, under the FR and the EOI case. A $35 \mathrm{~mW}-452 \mathrm{~nm}$ master blue laser diode, biased at $68 \mathrm{~mA}$ and $31^{\circ} \mathrm{C}$, is used to optically inject a slave blue laser diode with same specifications but biased at $61 \mathrm{~mA}$ and $20^{\circ} \mathrm{C}$. On the other hand, an $18 \mathrm{~mW}-660 \mathrm{~nm}$ master red laser diode is biased at $108 \mathrm{~mA}$ and $20^{\circ} \mathrm{C}$ is used to externally inject a slave red laser diode with the same specifications and biased at $100 \mathrm{~mA}$ and $34^{\circ} \mathrm{C}$. These biasing conditions of current and temperature are the optimized ones to achieve identical lasing wavelengths of both the laser diodes for efficient optical injection. Similar to the SIL, EOI spectrums also demonstrate an improvement of the peak power and reduction in the spectral linewidth of the dominant mode, and suppression of the side modes, thereby improving the SMSR. In this case, the peak power is increased by $35 \%$ in both the laser diodes compared to the respective FR cases, as shown in Figure 4 (c) and (d). These observations are the signatures of external injection locking occurring in the system. However, not much improvement in the modulation bandwidth (not shown) is observed in this case compared to FR, suggesting weak locking efficiency. In other words, we postulate that Eqn. (1) is probably satisfied in this case while the phase condition is not rigorously fulfilled. The authors strongly believe that employment of a polarization controller in the system to lock the polarization of both the laser diodes should enable substantial improvement in the locking efficiency and hence the modulation bandwidth of the laser, a clear signature of proper EIL. Unfortunately, this component was unavailable in free space for the blue and the red color region at the time of the experiment. Therefore, the authors resorted to using EOI in represent this work rather than EIL.

Next, the effect of IR (ratio of the master laser power to the slave laser power estimated at the slave front facet) on the external optically injected slave laser diode is investigated in Figure 5. Here, the diodes are operated at the aforementioned fixed injection currents, and an optical attenuator is employed at the front end of the master laser to control the power and hence the IR. A general trend of improved peak power and the spectral linewidth on increasing IR is apparent from the figure, for both the laser diodes types. Besides, the measured values of both the parameters are found to be better than the FR case at all IR. In view of the blue laser diode, shown in Figure 5(a), an increase in the IR up to $\sim 0.7 \mathrm{~dB}$ improved the peak power by $\sim 1.7$ times and reduced the spectral linewidth from $\sim 220 \mathrm{pm}$ to $\sim 78 \mathrm{pm}(\sim 2.8$ times reduction), displaying a minimum linewidth of $\sim 70 \mathrm{pm}$ at $-0.6 \mathrm{~dB}$ IR. Moreover, the performance reached a steady state at -0.6 
$\mathrm{dB}$ IR without further improvement in the characteristics. This is attributed to increasing IR which improves the EOI efficiency and hence a stronger signature of possible locking. On the other front, from Figure 5 (b) that summarizes the results of the red EOI laser diode, a steady behavior of peak power and linewidth reduction is noticed with increasing IR. A maximum improvement of $\sim 1.4$ times in peak power and $\sim 1.6$ times $(\sim 140 \mathrm{pm}$ to $\sim 87 \mathrm{pm})$ in linewidth, is noted at IR values of -5.0 and $-1.0 \mathrm{~dB}$, respectively. While the two regions, linewidth reduction $-11<\mathrm{IR}<-1.0 \mathrm{~dB}$ and saturation $\mathrm{IR}>-1.0 \mathrm{~dB}$, are noticeable in the red EOI laser diode, the latter region bounds are also found to be the onset of blue EOI laser diode as well, with the former region covers the range of $-5.0 \mathrm{~dB}<\mathrm{IR}<-0.6 \mathrm{~dB}$. These trend of optical injection dynamics are similar to that of the injection locked laser diodes reported in the literature [23], and suggests consistency in our measurements and further affirming our attribution of EOI as a signature of EIL. Moreover, Figure 5 also indicates another possible further transition into an unstable region for $>0.7 \mathrm{~dB}$ (blue) and $>1.3 \mathrm{~dB}$ (red), with inferior performance, as observed in literature [23-25]. On the whole, comparing the results of SIL and EOI, we found that the linewidth of blue (red) laser exhibited $\sim 80(\sim 90)$ pm value under selfinjection and $\sim 78(\sim 100) \mathrm{pm}$ value under external-injection, and at a fixed IR of $-0.7 \mathrm{~dB}$ and $-1.25 \mathrm{~dB}$, respectively. This suggests comparable performance from both the system. However, it is to be noted that at low current injections, SIL is found to be superior.
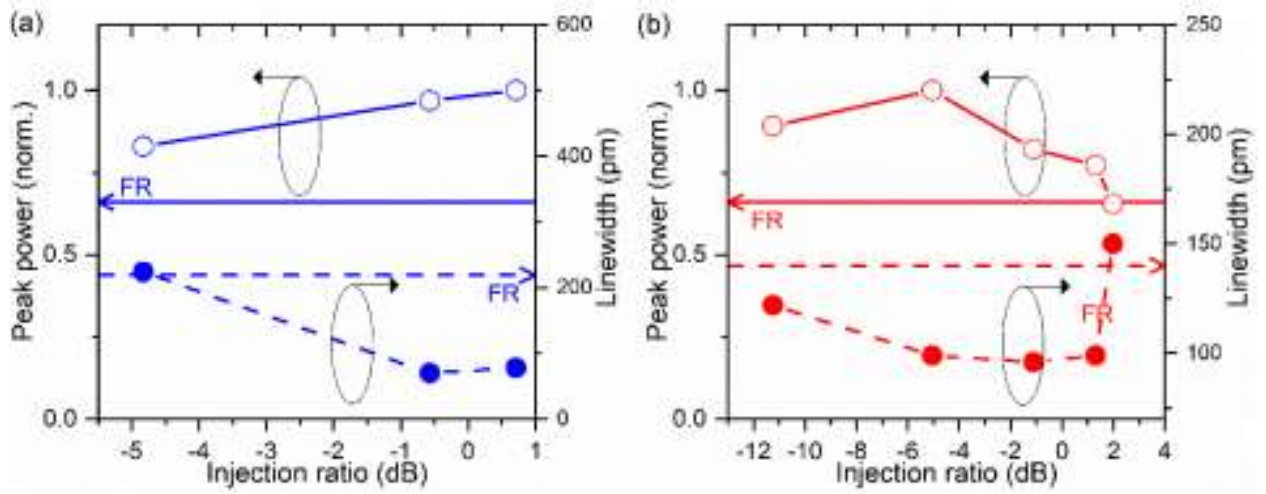

Figure 5: Variation of the peak power and spectral linewidth as a function of external optical injection ratio for (a) blue and (b) red laser diodes. The results of the FR case are also plotted for comparison purpose. The bias conditions of the EOI system for both the colors follows that of Fig. 4.

\subsection{Stability Performance}

A short-term stability test is performed on the EOI blue and red laser diode system for 5 minutes and the data were recorded at a 1-minute interval, and the results are shown in Figure 6. Firstly, the peak power for the blue and red lasers, shown in Figure 6(a) is found to have a normalized variation of $\pm 20 \%$ and $\pm 12 \%$, respectively, while the corresponding spectral linewidths (Figure 6 (b)) exhibits a small variation of $\pm 5 \mathrm{pm}$ and $\pm 10 \mathrm{pm}$. On the other hand, the peak wavelength shows a very stable performance with zero fluctuation of the EOI domination mode at $452.68 \mathrm{~nm}$ and $660.10 \mathrm{~nm}$ for blue and red laser diodes, respectively. This indicates that the EOI system is quite stable, similar to the SIL system since this is a crucial requirement for practical applications. 
(a)

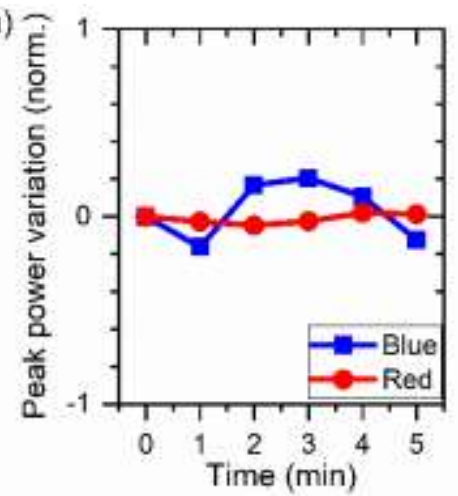

(b)

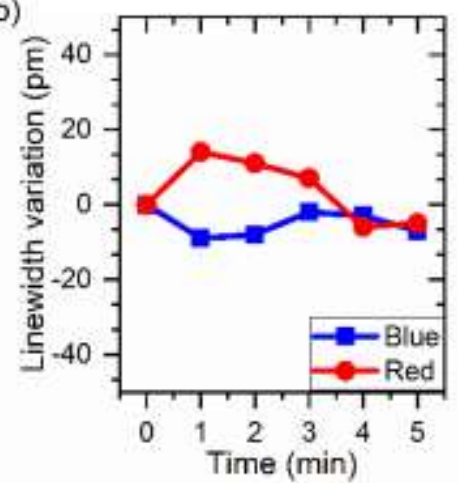

(c)

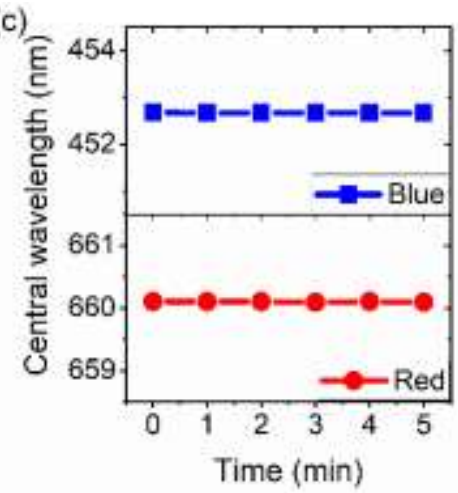

Figure 6: Short-term stability test for blue and red lasers under EOI showing (a) peak power, (b) linewidth variation, and (c) peak wavelength. The values are taken for 5 minutes at a 1-minute interval. The linewidth of both the laser diodes has been normalized with respect to the first value to show a better comparison. The bias conditions of both the color systems follow that of Fig. 5 .

\section{Visible Light Communication}

In this section, we discussed the OOK-NRZ transmission experiments performed, firstly, on the FR diode lasers and then on the optically injected laser system. We started with analyzing FR laser diode transmission performance with low sensitivity but large bandwidth ultra-high-speed photodiode (UPD) and employing an in-house fabricated laser diode mount, which significantly improved the data transmission rates of the system. Next, we examined the OOK-NRZ performance of SIL and EOI systems by comparing with the FR laser counterparts by employing a highly sensitive but low bandwidth APD. We resorted to this option and compromised on the photodiode bandwidth owing to small usable power ( 4-7\%) of the optically injected systems. Hence, in this case, the high sensitivity of the photodiode was crucial for the analysis. However, it is to be noted that the output power of these systems could be significantly enhanced by the optimized alignment of the components via incorporation of piezo three-axis translation stages and engaging high power laser diodes. Moreover, taking the usable output power from the rear un-coated facet of the slave (self-injection locked) laser diode in the EOI (SIL) system, if accessible, is another promising alternative. Thus, we utilized UPD along with a driver amplifier (Tektronix PSPL5865) in the case of the FR laser diode OOK-NRZ transmission, and APD for SIL and EOI laser diode configurations.

\subsection{OOK-NRZ transmission on a free-running laser diode}

A straightforward VLC $30 \mathrm{~cm}$ link for an FR laser diode is initially investigated, wherein a customized inhouse laser mount with reduced parasitic capacitance (i.e., large bandwidth) is utilized. Moreover, a biastee $(0.1-6 \mathrm{GHz})$ is employed at the transmitter side for direct modulation purpose with a UPD at the receiver side. Considerably higher modulation bandwidths of $\sim 2.0$ and $\sim 2.5 \mathrm{GHz}$ for the blue and the red laser diodes, respectively, are measured from this system, which is double in value compared to the $\sim 0.8-$ $1 \mathrm{GHz}$ measured from the standard laser mount employed in the optically injected systems. This enabled successful error-free OOK-NRZ transmission of up to 3.5 (4.5) $\mathrm{Gb} / \mathrm{s}$ for the blue (red) FR laser diodes, respectively, as depicted in Figure 7 [27]. Note that, the data rate for the red laser diode is the best value reported so far using OOK, to the author's knowledge. Moreover, it is noteworthy to mention here that at these highest data rates, the measured bit-error-rate (BER) was $\sim 2.5 \times 10^{-3}$ and $\sim 0.8 \times 10^{-3}$, which are well below the forward error correction (FEC) limit of $3.8 \times 10^{-3}$, indicating further room of data rate enhancement. 


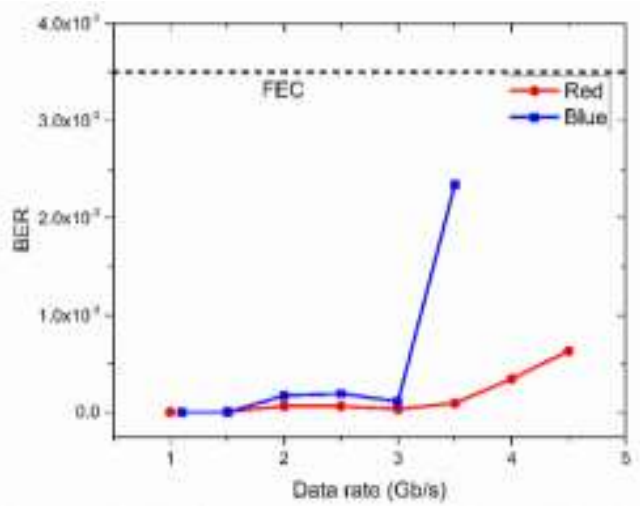

Figure 7: Bit-error rate as a function of the data rate for the $30 \mathrm{~cm}$ VLC link where blue and red laser diodes under FR configurations are employed. In this case, UPD and in-house laser diode mount are utilized in the system. The injection currents were 160 and $100 \mathrm{~mA}$ for blue and red laser diodes, respectively.

\subsection{OOK-NRZ transmission employing a SIL blue and red laser diode.}

Next, we performed the OOK-NRZ transmission experiments utilizing the setup of Figure 1(a) and employing APD at the receiver. The results of the effect of data rate on the BER of the system is illustrated in Figure 8 (a) and (b) for blue and red laser diodes, respectively, alongside the FR cases for comparison purpose. A maximum of $2.0 \mathrm{~Gb} / \mathrm{s}$ data rate is demonstrated in both cases, limited by the APD as well as laser mount bandwidth $(\sim 1 \mathrm{GHz})$ as both the laser diodes exhibited $\sim 1.0 \mathrm{GHz}$ modulation bandwidth in the system. Notice that both SIL and FR laser diodes demonstrated comparable performance, with a slight improvement in the former case at high data rates. Besides, a clear eye diagram at $2.0 \mathrm{~Gb} / \mathrm{s}$ of the SIL and the FR laser diodes, shown in the insets of Figures 8 (a) and (b), displays an error-free transmission. The authors' strongly believes that the limitation of APD bandwidth possibly have restricted observation of further improvement from the SIL system and attributed this slight improvement in part to a slight increase in the integrated power of the system, and partly to reduced system noise, under SIL, which has been observed previously [24]. A clear distinction in the transmission performance should be possible if phase modulation schemes were to be employed, as it is well known that injection locking substantially reduces the intensity as well as phase noise of the SIL laser system.
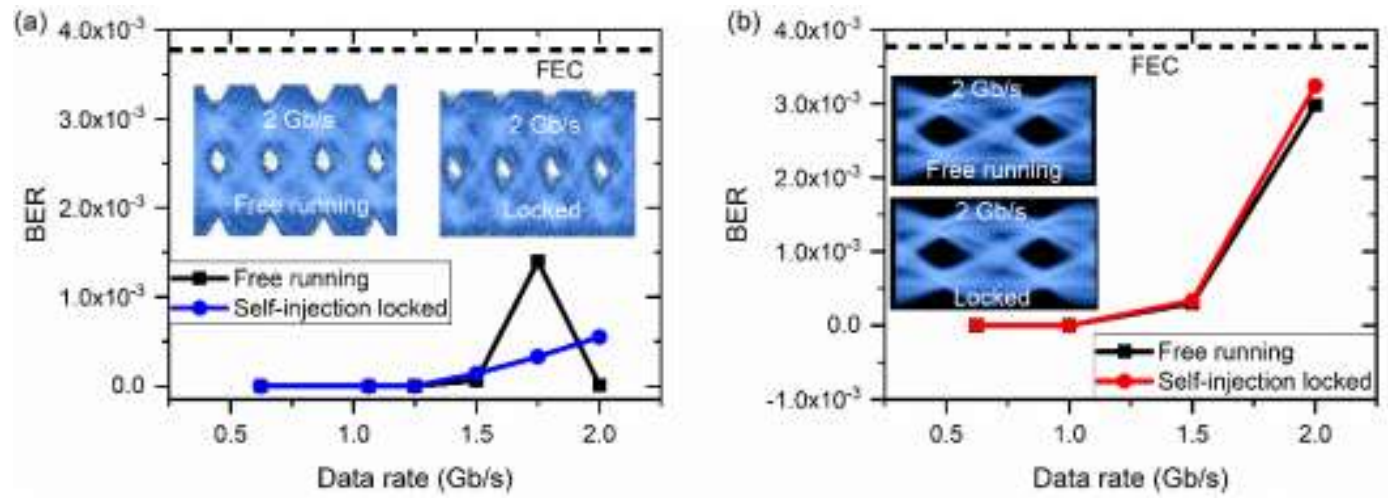

Figure 8: Bit-error-rate of the (a) blue and (b) red laser diodes at different data rates in the SIL laser diode VLC link. The eye diagram of the maximum data rate in each case is shown in the respective insets for the FR as well as SIL cases. In this case, APD and standard laser diode mount are utilized in the system 


\subsection{OOK-NRZ transmission employing EOI red laser diode}

Lastly, we performed the OOK-NRZ transmission experiments on the EOI red laser diode and compared the BER at different data rates alongside the FR case, and summarized in Figure 9. In this case, as well, the $-3 \mathrm{~dB}$ cut-off frequency of the FR red laser diode was measured to be $\sim 1.0 \mathrm{GHz}$ due to the employment of the standard laser diode mount. For smaller data rates of up to $1.6 \mathrm{~Gb} / \mathrm{s}$ the performance of EOI and the FR red laser diode was found to be similar, reaching a BER value of $\sim 1.0 \times 10^{-8}$. However, on increasing the data rate further, the EOI laser system showed better performance, exhibiting a BER of $\sim 4.0 \times 10^{-5}$ at 1.8 $\mathrm{Gb} / \mathrm{s}$ and $\sim 5.0 \times 10^{-3}$ at $2.0 \mathrm{~Gb} / \mathrm{s}$. On the other hand, the corresponding BERs of the FR case was measured to be $\sim 4.0 \times 10^{-3}$ and $\sim 1.2 \times 10^{-2}$. This significant improvement in the BER in case of EOI at high data rates, with error-free transmission up to $1.8 \mathrm{~Gb} / \mathrm{s}$ is attributed to reduced noise of the system in the presence of optical feedback compared to the FR case since EOI showed the signatures of the injection locking thereby exhibiting soft locking characteristics.

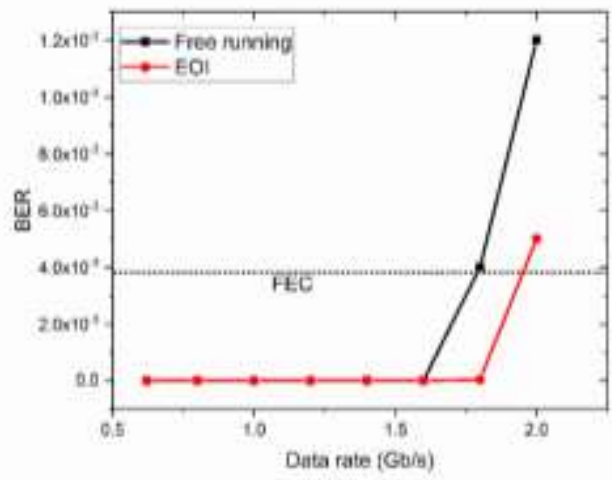

Figure 9: Bit-error-rate of the red laser diode at different data rates in an EOI configuration $30 \mathrm{~cm}$ VLC link for an injection current of $115 \mathrm{~mA}$ and $190 \mathrm{~mA}$ for the slave and master laser diodes respectively. In this case, APD and standard laser diode mount are utilized in the system

\section{Conclusion}

In summary, blue and red laser diodes were investigated employing SIL and EOI schemes. $~ 1.4$ and $~ 1.1$ times improvement in the modulation bandwidth and $\sim 6.5$ and $\sim 3.2$ times reduction in the spectral linewidth was noted from SIL blue and red laser diodes, respectively. Besides, comparing the performance across various cavity length SIL systems, the $19 \mathrm{~cm}$ short cavity length configuration displayed superior performance by a factor of $\sim 1.1-1.3$ in bandwidth, linewidth, and side-mode-suppression-ratio improvement. For the EOI system, a comprehensive analysis of the effect of injection ratio on the system performance showed $~ 1.6-2.0$ times improvement in both, peak power and the linewidth, while the latter linewidths exhibited values as small as $\sim 70 \mathrm{pm}$ and $\sim 87 \mathrm{pm}$ for blue and red laser diodes, respectively. Finally, transmission experiments based on direct OOK-NRZ modulation-scheme displayed better performance of up to $2 \mathrm{~Gb} / \mathrm{s}$ from the optically injected system compared to the FR case and are found to be limited by the bandwidth of the systems. Moreover, up to 3.5 and $4.5 \mathrm{~Gb} / \mathrm{s}$ data rates were also demonstrated in FR blue and red laser diodes on the in-house laser diode mount based system, thereby exhibiting a collective $8 \mathrm{~Gb} / \mathrm{s}$ data capacity should multiplexed system is implemented.

\section{Acknowledgment}

M. H. M. S, M. A. S, and M. Z. M. K acknowledge the support by Deanship of Research, King Fahd University of Petroleum and Minerals, through KAUST004 grant. The other authors acknowledges the partial support by King Abdulaziz City for Science and Technology (KACST), (grant no. KACST TIC R2- 
FP-008); partial support from King Abdullah University of Science and Technology (KAUST) baseline funding (grant nos. BAS/1/1614-01-01, KCR/1/2081-01-01, and GEN/1/6607-01-01); and KAUSTKFUPM Special Initiative (KKI) Program (REP/1/2878-01-01).

\section{References}

[1] J. R. D. Retamal et al., "4-Gbit/s visible light communication link based on 16-QAM OFDM transmission over remote phosphor-film converted white light by using blue laser diode," Optics Express, vol. 23, no. 26, p. 33656, Dec. 2015.

[2] "Wireless future drives microwave photonics," Nature Photonics, vol. 5, no. 12, pp. 724-724, Dec. 2011.

[3] H. Elgala, R. Mesleh, and H. Haas, "Indoor optical wireless communication: potential and state-of-the-art," IEEE Communications Magazine, vol. 49, no. 9, pp. 56-62, Sep. 2011.

[4] R. Perez-Jimenez, J. Rufo, C. Quintana, J. Rabadan, and F. J. Lopez-Hernandez, "Visible light communication systems for passenger inflight data networking," in 2011 IEEE International Conference on Consumer Electronics (ICCE), Las Vegas, NV, USA, 2011, pp. 445446 .

[5] N. Kumar, N. Lourenco, D. Terra, L. N. Alves, and R. L. Aguiar, "Visible light communications in intelligent transportation systems," in 2012 IEEE Intelligent Vehicles Symposium, Alcal de Henares, Madrid, Spain, 2012, pp. 748-753.

[6] I. Takai, T. Harada, M. Andoh, K. Yasutomi, K. Kagawa, and S. Kawahito, "Optical Vehicle-to-Vehicle Communication System Using LED Transmitter and Camera Receiver," IEEE Photonics Journal, vol. 6, no. 5, pp. 1-14, Oct. 2014.

[7] H. M. Oubei, C. Li, K.-H. Park, T. K. Ng, M.-S. Alouini, and B. S. Ooi, "23 Gbit/s underwater wireless optical communications using directly modulated $520 \mathrm{~nm}$ laser diode," Optics Express, vol. 23, no. 16, p. 20743, 2015.

[8] H. Chun et al., "LED Based Wavelength Division Multiplexed $10 \mathrm{~Gb} / \mathrm{s}$ Visible Light Communications," Journal of Lightwave Technology, vol. 34, no. 13, pp. 3047-3052, Jul. 2016.

[9] A. M. Khalid, G. Cossu, R. Corsini, P. Choudhury, and E. Ciaramella, "1-Gb/s Transmission Over a Phosphorescent White LED by Using Rate-Adaptive Discrete Multitone Modulation," IEEE Photonics Journal, vol. 4, no. 5, pp. 1465-1473, Oct. 2012.

[10] G. Ghione, Semiconductor Devices for High-Speed Optoelectronics. Cambridge: Cambridge University Press, 2009.

[11] B. Fahs et al., "A 6-m OOK VLC Link Using CMOS-Compatible p-n Photodiode and Red LED," IEEE Photonics Technology Letters, vol. 28, no. 24, pp. 2846-2849, Dec. 2016.

[12] F. M. Wu et al., "Performance Comparison of OFDM Signal and CAP Signal Over High Capacity RGB-LED-Based WDM Visible Light Communication," IEEE Photonics Journal, vol. 5, no. 4, pp. 7901507-7901507, Aug. 2013.

[13] J. J. Wierer, J. Y. Tsao, and D. S. Sizov, "Comparison between blue lasers and light-emitting diodes for future solid-state lighting: Comparison between blue lasers and light-emitting diodes," Laser \& Photonics Reviews, vol. 7, no. 6, pp. 963-993, Nov. 2013.

[14] S. Watson et al., "Visible light communications using a directly modulated $422 \mathrm{~nm}$ GaN laser diode," Optics Letters, vol. 38, no. 19, p. 3792, Oct. 2013.

[15] Y. C. Chi, D. H. Hsieh, C. T. Tsai, H. Y. Chen, H. C. Kuo, and G. R. Lin, "450-nm GaN laser diode enables high-speed visible light communication with 9-Gbps QAM-OFDM," Opt Express, vol. 23, no. 10, pp. 13051-13059, 2015.

[16] H.-H. Lu et al., " $45 \mathrm{~Gb} / \mathrm{s}$ PAM4 transmission based on VCSEL with light injection and optoelectronic feedback techniques," Optics Letters, vol. 41, no. 21, p. 5023, Nov. 2016.

[17] P. S. Donvalkar, A. Savchenkov, and A. Matsko, "Self-injection locked blue laser," Journal of Optics, vol. 20, no. 4, p. 045801 , Apr. 2018.

[18] M. A. Shemis et al., "L-Band Quantum-dash Self-Injection Locked Multiwavelength Laser Source for Future WDM Access Networks," IEEE Photonics Journal, vol. 9, no. 5, pp. 1-7, Oct. 2017.

[19] C.-Y. Li et al., "16 Gb/s PAM4 UWOC system based on 488-nm LD with light injection and optoelectronic feedback techniques," Optics Express, vol. 25, no. 10, p. 11598, 2017.

[20] C.-Y. Li et al., "16 Gb/s PAM4 UWOC system based on 488-nm LD with light injection and optoelectronic feedback techniques," Optics Express, vol. 25, no. 10, p. 11598, May 2017.

[21] H.-H. Lu et al., "A 56 Gb/s PAM4 VCSEL-Based LiFi Transmission With Two-Stage Injection-Locked Technique," IEEE Photonics Journal, vol. 9, no. 1, pp. 1-8, Feb. 2017.

[22] T. Hosoya, M. Miranda, R. Inoue, and M. Kozuma, "Injection locking of a high power ultraviolet laser diode for laser cooling of ytterbium atoms," Review of Scientific Instruments, vol. 86, no. 7, p. 073110, Jul. 2015.

[23] M. H. M. Shamim et al., "Investigation of Self-Injection Locked Visible Laser Diodes for High Bit-Rate Visible Light Communication," IEEE Photonics Journal, vol. 10, no. 4, pp. 1-11, Aug. 2018.

[24] M. H. M. Shamim et al., "Enhanced performance of $450 \mathrm{~nm} \mathrm{GaN}$ laser diodes with an optical feedback for high bit-rate visible light communication," in Conference on Lasers and Electro-Optics, San Jose, California, 2018, p. JTu2A.29.

[25] J. Ohtsubo, "Theory of Optical Feedback in Semiconductor Lasers," in Semiconductor Lasers, vol. 111, Berlin, Heidelberg: Springer Berlin Heidelberg, 2013, pp. 75-101.

[26] L. A. Coldren, S. W. Corzine, and M. L. Mašanović, Diode Lasers and Photonic Integrated Circuits: Coldren/Diode Lasers 2E. Hoboken, NJ, USA: John Wiley \& Sons, Inc., 2012.

[27] C. Lee et al., "4 Gbps direct modulation of $450 \mathrm{~nm}$ GaN laser for high-speed visible light communication," Optics Express, vol. 23, no. 12, p. 16232, Jun. 2015. 